\title{
Avaliação de mapas conceituais e estrutura de conhecimento em acadêmicos de medicina.
}

\section{Evaluation of concept maps and knowledge structure of medicine undergraduate students}

\author{
Claudia Yamada Utagawa ${ }^{1} 2$ | claudia.utagawa@foa.org.br \\ Rejane Ribeiro ${ }^{2}$ \\ Maria Lucia Bianconi²
}

\section{RESUMO}

Uma bem elaborada estrutura de conhecimento é um dos requisitos fundamentais para que um indivíduo consiga aplicar na prática o que foi aprendido, segundo a visão cognitivista. Para isso, os novos conhecimentos devem ser integrados a conhecimentos previamente existentes. Na formação médica, esse conhecimento bem estruturado contribuiria na prática reflexiva e na tomada de decisões. Um dos problemas enfrentados, entretanto, é como avaliar essa estrutura de conhecimento. Na maioria das vezes o processo avaliativo concentra-se no conteúdo e nas habilidades procedimentais e não na qualidade dessa estruturação. Uma das ferramentas que poderia ser útil seria o mapa conceitual (MC), uma representação gráfica de conceitos e de relações entre conceitos em um determinado domínio de saber. O objetivo desse trabalho foi verificar a validade da utilização dos MC na avaliação da estruturação de conhecimento de alunos de graduação em um Curso de Medicina utilizando a metodologia estrutural. Vinte e três alunos participaram do projeto confeccionando mapas conceituais individuais pré e pós intervenção da pesquisadora, sobre o tema anemia. Os primeiros mapas (Mapa 1) foram confeccionados, após uma breve instrução. Num segundo encontro, os alunos receberam seus mapas avaliados apontando erros conceituais e de confecção de mapas, com sugestão de aprimoramento. Para o segundo mapa (Mapa 2), os alunos deveriam consultar livros textos para auxiliar a construção, observando as sugestões dadas. Os mapas foram pontuados pela metodologia estrutural (NOVAK e GOWIN, 1987) e os resultados revelaram que as pontuações dos Mapas 2 foram significativamente maiores que as dos Mapas 1. Contribuíram para maior pontuação o número de proposições, de hierarquia e de relações cruzadas entre conceitos. Esses achados demonstram que a metodologia estrutural poderia ser utilizada como avaliação preliminar da estrutura de conhecimento do aluno. Deve-se observar, entretanto, que a confecção e avaliação dos mapas necessitam de alto comprometimento tanto do aluno como do professor/tutor, por necessitarem de maior tempo despendido e por ser totalmente individualizada.

Palavras-chave: Mapas Conceituais, Avaliação, Estudantes de Medicina

UniFOA - Centro Universitário de Volta Redonda, Volta Redonda

2 IBqM-UFRJ - Instituto de Bioquímica Médica/ Universidade Federal do Rio de Janeiro, Rio de Janeiro 


\section{ABSTRACT}

According to the cognitive point of view, a well elaborate knowledge structure is an essential requirement for an individual to apply whatever is learned. In order to achieve that, new knowledge must be integrated to the old ones. In the medical schooling, this well-structured knowledge can contribute to a reflexive practice and to decision making. However, one of the problems is related to the evaluation of this structure of knowledge. Most of the time, the evaluation do not assess the quality of this new structure but focus the theoretical contents and in procedural skills. Concept maps (CM), a graphical representation of concepts and the relationship between them could be useful tools in determined fields of knowledge. The objective of this work was to validate the CM utilization in the assessment of the structure of knowledge of undergraduate students of a Medicine School by using the structural methodology. Twenty-three students participated on the project producing individual CM on the theme anemia, previously and after an intervention of the researcher. The first maps (Map 1) were created after a brief instruction. In a second meeting, the students received their corrected maps where conceptual errors as well as mistakes related to map construction were pointed together with suggestions for improvement. The students should search textbooks and pay attention to the suggestions in order to construct the second map (Map 2). The maps were punctuated according to the structural methodology (NOVAK \& GOWIN, 1987), and the results revealed that Maps 2 where significantly improved with better punctuation than Maps 1. The number of propositions, hierarchical structure, and cross-links contributed to a higher punctuation. These findings show that the structural methodology could be used as a preliminary evaluation of the student's structure of knowledge. Nevertheless, it is important to point out that both the production and evaluation of concept maps require a high commitment from the student and the professor/tutor since it is time consuming and an entirely individualized process.

Keywords: Conceptual maps, Evaluation, Medicine students.

\section{INTRODUÇÃO}

Para se tornar um especialista em qualquer área de conhecimento, um indivíduo necessita aprender conteúdos específicos e procedimentos para, então, compreender como e quando o conhecimento poderá ser aplicado. Um dos passos decisivos para a incorporação desses conhecimentos depende de uma bem elaborada e integrada estrutura de conhecimento. Segundo os cognitivistas, o aprendizado não pode ocorrer somente de maneira mecânica por memorização de novos fatos e conceitos. É essencial que as informações sejam assimiladas integrando-se a uma estrutura de conhecimento já existente, tornando-se, portanto, significativa (AUSUBEL, 1968).

Em relação ao conhecimento médico, vários pesquisadores salientam a importância de se encorajar seu uso baseado em práticas que incentivem o julgamento crítico, a prática reflexiva, a resolução de problemas e a tomada de decisões. (MAUDSLEY e STRIVENS, 2000; WEST et al., 2002). Um dos desafios na educação médica, entretanto, está em como avaliar a estruturação desse conhecimento pois, na maioria das vezes, o processo avaliativo concentra-se no conteúdo e nas habilidades procedimentais e não na qualidade dessa estruturação.

Estudos que remontam 35 anos apontam que uma das ferramentas que poderiam contribuir com o processo de estruturação e avaliação do conhecimento são os mapas conceituais (MC). Os MC foram desenvolvidos em 1972 por Joseph Novak e seus colegas da Universidade de Cornell. Eles se baseiam na Teoria da Aprendizagem Significativa (AUSUBEL, 1968). Segundo Novak e Cañas (2008), “mapas conceituais são ferramentas gráficas para organizar e representar conhecimento. Eles incluem conceitos, usualmente dentro de círculos ou caixas de algum tipo e a indicação da relação entre os conceitos é realizada por linhas que ligam esses conceitos”. A estruturação do conhecimento na mente humana tende a seguir uma estrutura hierárquica, na qual as ideias mais abrangentes incluem proposições, conceitos e dados menos inclusivos e mais diferenciados, que podem ser usados na construção dos mapas. Os mapas podem demonstrar o nível de aprofundamento de compreensão 
daquele que constrói essas representações gráficas (ROBERTS, 1999). Alguns autores têm salientado a utilidade dos MC em educação e apontaram que, no processo inicial de construção de mapas dentro de um dado campo de conhecimento, a ferramenta auxilia os alunos a clarificar sua compreensão sobre o tópico e facilita a retenção do conhecimento. Também tem utilidade para que os professores/tutores possam prover feedback aos seus alunos/ aprendizes. Salientam também a possibilidade de se poder avaliar, através dos mapas, a compreensão do aluno, seu grau de aprofundamento e sua estruturação cognitiva (ROBERTS, 1999; WEST et al., 2002; SRINIVASAN et al., 2008; DALEY e TORRE, 2010).

\section{OBJETIVO}

O objetivo desse trabalho foi verificar a validade da utilização dos MC na avaliação da estruturação de conhecimento de alunos de graduação em um Curso de Medicina utilizando a metodologia estrutural.

\section{METODOLOGIA}

Alunos de graduação do Curso de Medicina a partir do $2^{\circ}$ ano de formação foram convidados a participar do projeto, realizado no Centro Universitário de Volta Redonda - UniFOA, num curso de capacitação sobre MC. O projeto foi submetido e aprovado pelo Comitê de Ética em Pesquisa dessa instituição. Todos os participantes assinaram um termo de consentimento livre e esclarecido e responderam um questionário para conhecimento de dados pessoais e conhecimento sobre MC. Um treinamento de 60 minutos sobre a confecção de mapas conceituais, segundo a metodologia de NOVAK e CAÑAS (2008) foi realizado. Após o treinamento, os participantes foram convidados a construir seus MC sobre o tema Anemia, já estudado durante a graduação. Os alunos confeccionaram seus primeiros mapas (MAPA 1) de maneira independente em uma folha de papel em branco A4 durante 30 minutos. Todos os MC foram codificados. Uma semana após, um novo encontro com os alunos foi agendado, onde cada aluno recebeu seu mapa de volta avaliado pela pesquisadora responsável, apontando erros conceituais e de metodologia de confecção. Após o esclarecimento de dúvidas dos alunos, foi solicitado que estudassem o tema abordado em livros textos e confeccionassem um novo mapa, chamado de MAPA 2. Os mapas 1 e 2 de cada aluno foram analisados através do modelo estrutural (NOVAK e GOWIN, 1987). Os dados obtidos foram inseridos em planilha Excel e analisados com o teste estatístico paramétrico ANOVA (Analysis of Variance).

\section{RESULTADOS}

Inscreveram-se no curso 25 alunos do curso de Medicina, entretanto, dois alunos não concordaram em participar do projeto. Vinte e três alunos confeccionaram seus pares de mapas, sendo 19 do sexo feminino e 4, do sexo masculino. A idade variou de 18 a 27 anos, com média de 21 anos. A maior parte dos participantes era aluno do $4^{\circ}$ período do curso de Medicina (60,8\%). Cinco alunos eram do $6^{\circ}$ Período (21,7\%), dois alunos do $7^{\circ}$ período $(8,7 \%)$ e os outros dois do $8^{\circ}$ e $9^{\circ}$ períodos. Quinze alunos $(65,2 \%)$ declararam desconhecer o que era um MC. O restante já tinha visto um MC, mas não sabia como confeccioná-lo.

Dos 23 pares de MC confeccionados, um par foi excluído, uma vez que não preenchia os critérios de construção de MC, conforme explicitado logo no início da capacitação. Os mapas confeccionados à mão foram inseridos no programa IHMC CmapTools para facilitar a avaliação que foi realizada a partir do método estrutural que consiste na soma de pontos de quatro categorias: proposições, níveis de hierarquia, presença de ligação entre conceitos e de exemplos. As categorias proposição e exemplos corretos recebiam 1 ponto. Cada nível de hierarquia, que consistia em relações de categorias superordinárias (mais gerais) para subordinadas (mais específicas) recebia 5 pontos. A relação cruzada entre conceitos é aquela que liga dois conceitos localizados em diferentes ramos de domínios de conhecimento no mapa, é o mais valorizados, recebendo 10 pontos para cada item correto. 
A partir dos resultados obtidos foi aplicado o teste estatístico ANOVA, assumindo um nível de significância $\alpha$ = 0,05 e comparando-se as médias e as variâncias dos M1 e dos M2. Para esta análise foi utilizado o programa Origin 5.0 (MicroCal, Llc). Os resultados dessa análise estão dispostos no gráfico 1 e na tabela 1. As figuras 1 e 2 representam um exemplo dos Mapas 1 e 2 confeccionados por um dos participantes.

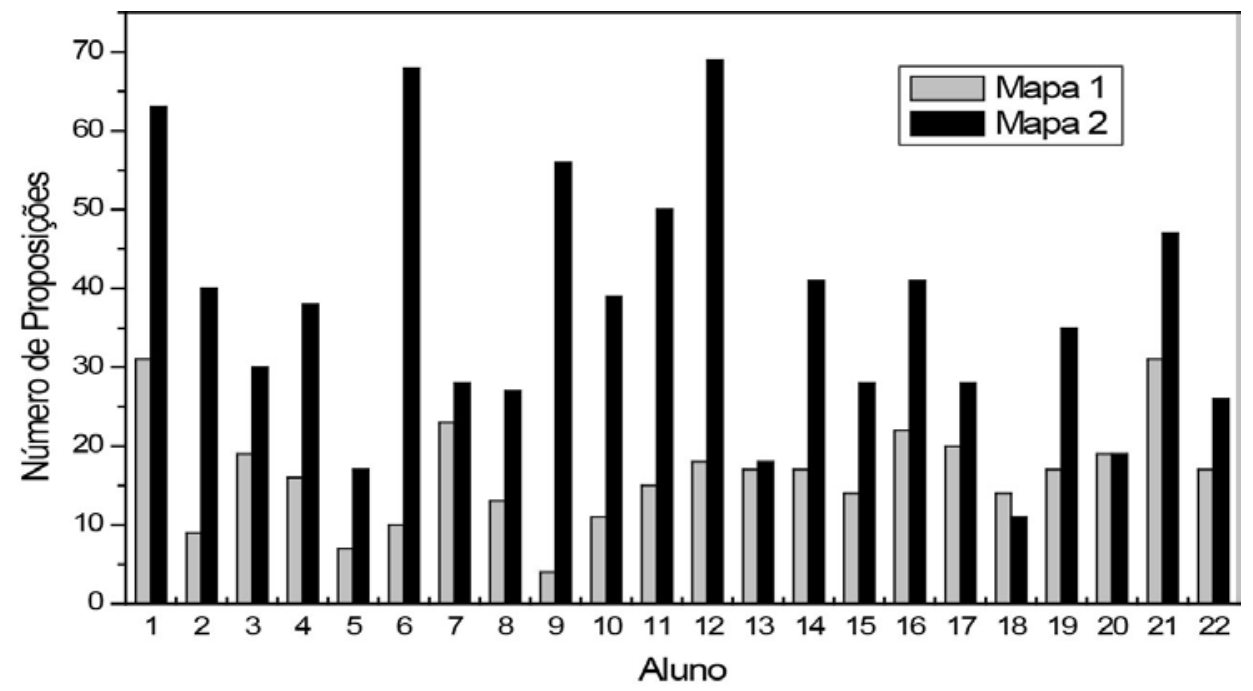

Gráfico 1. Comparação entre as pontuações obtidas para o número de proposições entre o mapa 1 e o mapa 2 para cada aluno.

\begin{tabular}{|c|c|c|c|c|}
\hline \multirow{2}{*}{ Categorias } & & \multirow{2}{*}{ Média \pm SD } & \multicolumn{2}{|c|}{ ANOVA } \\
\hline & & & $\mathbf{F}$ & $\mathbf{p}$ \\
\hline \multirow{2}{*}{ Proposições } & Mapa 1 & $16,5 \pm 6,6$ & 30,22872 & 0,00000 \\
\hline & Mapa 2 & $37,2 \pm 16,4$ & & \\
\hline \multirow{2}{*}{ Hierarquia } & Mapa 1 & $2,1 \pm 0,6$ & 17,62694 & 0,00014 \\
\hline & Mapa 2 & $3,0 \pm 0,7$ & & \\
\hline \multirow{2}{*}{ Relação cruzada entre conceitos } & Mapa 1 & $0,5 \pm 1,0$ & 7,99932 & 0,00714 \\
\hline & Mapa 2 & $2,4 \pm 3,0$ & & \\
\hline \multirow{2}{*}{ Exemplos } & Mapa 1 & $1,7 \pm 2,2$ & 2,77658 & 0,10309 \\
\hline & Mapa 2 & $3,3 \pm 3,9$ & & \\
\hline \multirow{2}{*}{ Pontuação total } & Mapa 1 & $34,0 \pm 15,5$ & 17,86836 & 0,00012 \\
\hline & Mapa 2 & $79,0 \pm 47,6$ & & \\
\hline
\end{tabular}

Tabela 1. Análise da pontuação geral dos mapas 1 e 2.

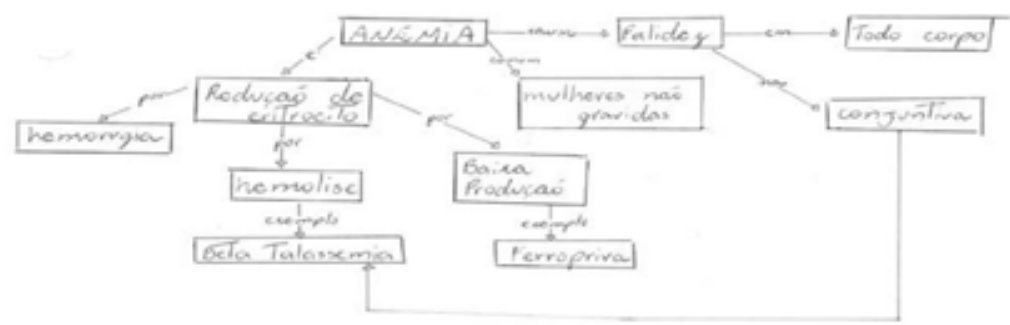

Figura 1. Mapa 1, realizado por um aluno durante a pré-intervenção. 


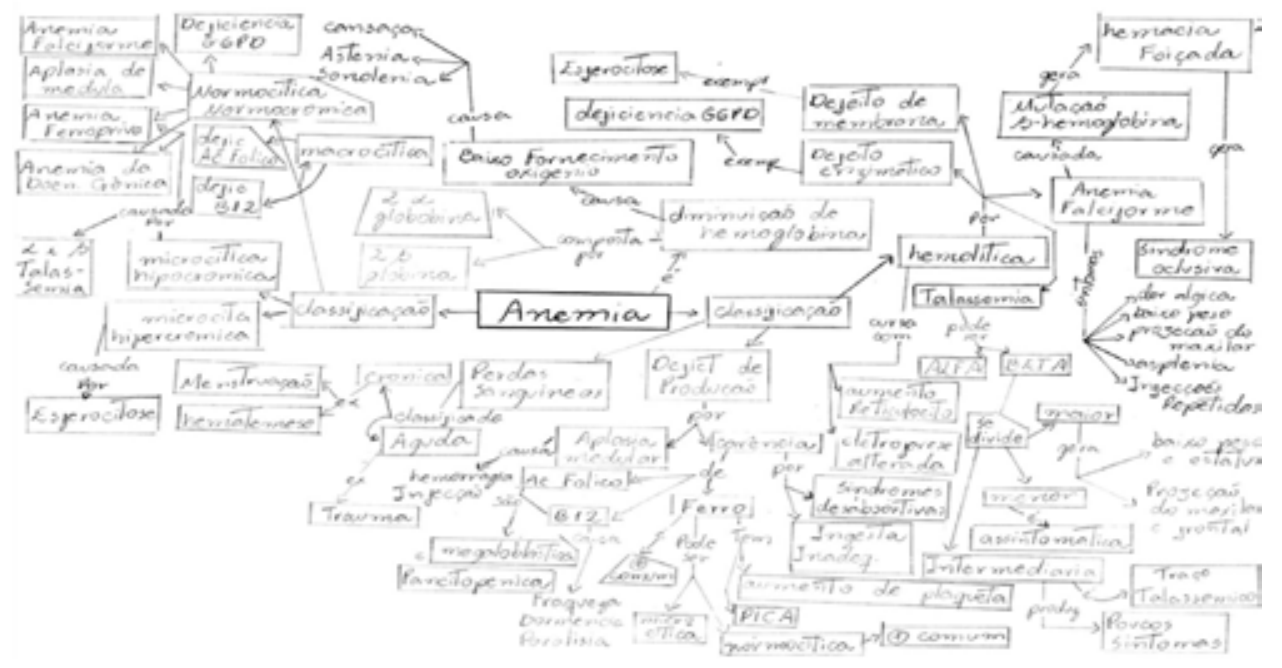

Figura 2. Mapa 2, realizado durante a pós intervenção, pelo mesmo aluno do exemplo da Figura 1.

\section{DISCUSSÃO TEÓRICA}

Não há muitos artigos em educação médica sobre a validade da utilização de MC para avaliação da estrutura de conhecimento em educação médica (NESBIT e WEST et al., 2002; NESBIT e ADESOPE, 2002; SRINIVASAN et al., 2008). Um dos motivos apontados é a dificuldade de mensuração desses mapas (WEST et al., 2002). A validade da avaliação dos MC é extremamente dependente de como os mapas são interpretados ou pontuados. Existem diversas metodologias para avaliação dos mapas. Uma das metodologias mais utilizadas para avaliação de mapas conceituais é a que se utiliza do método estrutural (NOVAK e GOWIN, 1984). Nesse método, o maior peso da pontuação baseia-se na organização de uma estrutura hierárquica do mapa, ligações dos conceitos e estabelecimento de relações cruzadas entre domínios de conhecimentos. A base teórica da metodologia está no aprofundamento do pensamento ou na compreensão requerida para a categoria focando na posição relativa de cada conceito do mapa. No presente trabalho verificou-se que os mapas 2 apresentaram maior pontuação final que os primeiros mapas confeccionados e que essa diferença era significativa, como demonstrado na tabela 1 . Se considerarmos todas as categorias, os itens: número de proposições, hierarquia e relação cruzada entre conceitos foram os que mais contribuíram de maneira significante para a maior pontuação final dos mapas. Esses achados estão de acordo com a literatura (NOVAK e GOWIN, 1984; WEST et al., 2002).

A aprendizagem significativa apresenta quatro princípios essenciais: a diferenciação progressiva, a reconciliação integrativa, a organização sequencial e a consolidação (MOREIRA e BUCHWEITZ, 1993; SOUZA e BORUCHOVITCH, 2010). A diferenciação progressiva implica na hierarquização de conceitos mais abrangentes até os mais específicos, que quanto mais integrados, refletem melhor estruturação de conhecimento, observada nos mapas, principalmente pelo número de ligação entre conceitos. A maioria dos participantes demonstrou avanços na hierarquia e no estabelecimento de ligação entre conceitos, demonstrando capacidade de realizar, pelo menos, os três primeiros princípios.

\section{CONCLUSÕES}

Podemos afirmar que houve uma diferença significativa entre os mapas 1 e 2 quando utilizada para análise a metodologia estrutural. Isso demonstra que, para uma análise preliminar da avaliação do aluno, essa metodologia poderia ser adotada. Algumas considerações, entretanto, devem ser feitas. Uma delas é que para que haja uma melhoria na qualidade desses mapas, deve-se existir o compromisso do docente/tutor, pois cada mapa deve ser avaliado individualmente, provendo o feedback necessário ao aprendiz e promovendo assim, a 
consolidação do conhecimento do mesmo. A adoção de MC para avaliação do conhecimento do aluno, portanto, demanda mais tempo para o processo, o que pode dificultar sua aplicação. Outra questão é a necessidade do empenho e colaboração do aluno que tem que ter bom domínio do processo de confecção de mapas, uma vez que essa falta de domínio pode prejudicar o resultado final da pontuação de seu mapa.

\section{REFERÊNCIAS BIBLIOGRÁFICAS}

AUSUBEL, D. P.; NOVAK, J. D.; HANESIAN, H. Psicologia Educacional. Rio de Janeiro: Interamericana, 1980.

DALEY B.J.; TORRE D.M. Concept maps in medical education: an analytical literature review. Med Educ. v. 44, n. 5, p. 440-448, 2010.

NOVAK, J.D.; CAÑAS, A. J. The Theory Underlying Concept Maps and How to Construct and Use Them. Technical Report IHMC CmapTools. Florida Institute for Human and Machine Cognition (IHMC), 2008, disponível em: < http://cmap.ihmc.us/Publications/ResearchPapers/TheoryUnderlyingConceptMaps.pdf>, acesso em julho 2010.

NOVAK, J.D.; GOWIN, D.B. Learning how to learn. New York: Cambridge University Press, 1984.

MAUDSLEY, G.; STRIVENS, J. “Science”, “critical thinking” and “competence” for Tomorrow's Doctors. A review of terms and concepts. Medical Education v. 34, p. 53-60, 2000.

MOREIRA, M. A.; BUCHWEITZ, B. Novas estratégias de ensino e aprendizagem: os mapas conceptuais e o Vê epistemológico. Lisboa: Plátano Edições Técnicas, 1993.

NESBIT, J.C.; ADESOPE, O.O. Learning with Concept and Knowledge Maps: A Meta-Analysis. Review of Education Research v. 76, n. 3, p. 413-448, 2006.

ROBERTS, L. Using concept maps to measure statistical understanding. Int. J. Math. Educ. Sci. Technol. v. 20, n. 5, p. 707-717, 1999.

SOUZA, N. A.; BORUCHOVITCH, E. Mapas conceituais: estratégia de ensino/ aprendizagem e ferramenta avaliativa. Educação em Revista v. 26, n. 3, p. 195-218, 2010.

SRINIVASAN, M.; MCELVANY, M., SHAY, J. M.; SHAVELSON, R. J.; WEST, D. C. Measuring Knowledge Structure: Reliability of Concept Mapping Assessment in Medical Education. Academic Medicine v. 83, n. 12, p. 1196-1203, 2008.

WEST, D. C.; PARK, J. K.; POMEROY, J. R.; SANDOVAL, J. Concept mapping assessment in medical education: a comparison of two scoring systems. Medical Education v. 36, p. 820-826, 2002. 